\title{
ASSESSMENT OF THE SEEV MODEL TO PREDICT ATTENTION ALLOCATION AT INTERSECTIONS DURING SIMULATED DRIVING
}

\author{
Nicholas D. Cassavaugh ${ }^{1}$, Alex Bos ${ }^{1}$, Cole McDonald ${ }^{1}$, Pujitha Gunaratne ${ }^{2}$ \& Richard W. Backs ${ }^{1}$ \\ ${ }^{1}$ Central Michigan University, Mount Pleasant, Michigan, USA \\ ${ }^{2}$ Toyota Motor Engineering \& Manufacturing North America, Ann Arbor, Michigan, USA \\ Email: cassa1nd@cmich.edu
}

\begin{abstract}
Summary: We attempted to model attention allocation of experienced drivers using the SEEV model. Unlike previous attempts, the present work looked at attention to entities (vehicles, signs, traffic control devices) in the outside world rather than considering the outside world as a unitary construct. Model parameters were generated from rankings of entities by experienced drivers. Experienced drivers drove a scenario that included a number of intersections interspersed with stretches of straight road. The intersections included non-hazard events. Eye movements were monitored during the driving session. The results of fitting the observed eye movement data to our SEEV model were poor, and were no better than fitting the data to a randomized SEEV model. A number of explanations for this are discussed.
\end{abstract}

\section{INTRODUCTION}

Visual scanning is the primary means of obtaining information from the world while driving (Cole, 1972). Understanding how drivers scan the environment and how experienced and novice drivers differ in their visual scanning are important areas for research. We set out to investigate attention allocation during driving in both novices and experienced drivers. Here we present the results of an initial investigation into using a computational model to model the attention allocation of experienced drivers. The present investigation focused on allocation of attention in non-hazard situations.

Wickens and colleagues (Wickens, Helleberg, Goh, Xu, \& Horrey, 2001) developed the SEEV model as a model of scanning behavior describing the probability that a given area of interest (AOI) will attract attention. SEEV refers to the Salience, Effort, Expectancy, and Value associated with a particular AOI. $P(A O I)$ is the probability of attending to a particular AOI. Salience (S) refers to the physical properties of events (e.g. their conspicuity or the likelihood that events will capture attention). Effort (Ef) refers to both the effort involved in reallocating attention to a new area of interest (AOI) and to the current mental workload engendered by the tasks at hand. Expectancy (Ex) refers to the expectancy of gaining information from an event or AOI, including the bandwidth and rate of information flow related to the event. Value refers to an objective measure of the value or cost of processing or failing to process information in a particular AOI and is represented as the product of Relevance and Priority $(\mathrm{R} * \mathrm{P})$. Equation 1 below illustrates the SEEV model in prescriptive terms (Horrey, Wickens, \& Consalus, 2006; Wickens, Goh, Helleberg, Horrey, \& Talleur, 2003; Wickens, et al., 2001).

$$
\mathrm{P}(\mathrm{AOI})=\mathrm{S} * \mathrm{Ex} *(\mathrm{R} * \mathrm{P})-\mathrm{Ef}
$$


While originally developed for use in aviation, the SEEV model has been used in other contexts. In particular Horrey and colleagues (Horrey, et al., 2006) applied the SEEV model to simulated driving and interactions with in-vehicle technologies. They compared head-up and head-down displays in driving. Horrey et al. (2006) found a strong fit between predicted and actual fixation, with $\mathrm{R}^{2}$ values above 0.90 . However, the authors only modeled two AOIs: the task display and the outside world (OW). Other work has extended the SEEV model to include dynamic salience to create the N-SEEV model (Steelman-Allen, McCarley, Wickens, Sebok, \& Bzostek, 2009). We wondered if the SEEV model could similarly be extended to model attention allocation to AOIs defined for entities within the OW.

We hypothesized that the SEEV model would successfully predict attention allocation to AOIs at intersections and that this would be evident in statistically significant regression analyses.

\section{METHOD}

\section{Participants}

Data from eight experienced participants are presented here. Of those, three were male and five were female. Their mean age was 32 years. All participants had been licensed for more than 8 years at the time of testing. We recruited participants via advertisements placed in local media and paid participants for their time.

\section{Method}

Apparatus. The driving simulator was a DriveSafety DS-600c with $180^{\circ}$ forward field of view. The simulator provides rear views using LCD panels as the side-view and rear-view mirrors. A motion base provides motion in pitch during acceleration and braking. The simulator cab is the forward passenger cabin of a Ford Focus. The simulator scenarios were created using HyperDrive 1.9.35 (DriveSafety).

Eye movements were recorded using SmartEye Pro 5.5 with three cameras mounted to the dashboard of the simulator cab. Video signals were captured from the forward image channel and routed through screen-capture software for later integration with eye movement data. MAPPS 2.1 (EyesDX) software was used to analyze eye movement data.

Simulator Scenario. The experimental scenario consisted of approximately $18 \mathrm{~km}$ of straight road with seven intersections. Several non-hazard events occurred at the intersections. These events were designed to draw attention without representing an imminent hazard. A list of the events is presented in Table 1 below. The manipulated SEEV parameter was varied between events by using vehicles rated at the extremes of that parameter by a separate sample of experienced participants who had completed a pilot survey. Sample ratings are presented in the results section and can be found in Table 3. 
Table 1. List of the non-hazard events presented at intersections

\begin{tabular}{cclc}
\hline Intersection \# & $\begin{array}{c}\text { SEEV Parameter } \\
\text { Manipulation }\end{array}$ & \multicolumn{1}{c}{$\begin{array}{c}\text { Intervening Vehicle } \\
\text { Behavior }\end{array}$} & $\begin{array}{c}\text { Traffic Light } \\
\text { Status }\end{array}$ \\
\hline 1 & N/A & Left turn from West lane & Red \\
2 & Expectancy & Right turn from South lane & Green \\
3 & Expectancy & Left turn from East lane & Red \\
4 & Value & Right turn from North lane & Green \\
5 & Value & Left turn from South lane & Red \\
6 & Expectancy & Right turn from West lane & Green \\
7 & Value & Left turn from North lane & Green \\
\hline
\end{tabular}

The posted speed limit was $64.4 \mathrm{~km} / \mathrm{h}$ (40 MPH). Speed limit signs were posted at several locations throughout the scenario. A vehicle followed the participant and flashed its headlights if the participant's speed dropped below $56.3 \mathrm{~km} / \mathrm{h}$ (35 MPH). A software governor limited speed $72.4 \mathrm{~km} / \mathrm{h}$ (45 MPH) or less. Total drive time was approximately 17 minutes.

In addition to the scripted non-hazard events at intersections, ambient traffic was present in the simulation. Ambient vehicles always traveled in the direction opposite the participant and posed no hazard.

Procedure. Participants were welcomed to the lab, informed of the purpose of the study and signed informed consent prior to participating. Following this, participants drove an adaptation scenario in the simulator. The purposes of this adaptation were to introduce the participants to the simulator, provide practice in driving it, and to provide an acclimation period to help in avoiding simulator sickness when driving the simulator later. After the adaptation, participants relocated to another room. We tested their visual acuity at near and far distances, collected demographic data and other information on driving behavior and experience.

Participants then returned to the simulator. During this period we created a profile for the eye tracking system and calibrated the system. Following this, participants drove the experimental scenario. After completing the driving scenario, we paid the participants, thanked them for their participation, and sent them on their way. The experiment took approximately one hour to complete.

\section{Analysis plan}

SEEV Models. We developed two different SEEV Models: a simplified model using only three AOIs (Dashboard, Looking Ahead, Other) as a comparison to the work of Horrey et al. (2006), and a more complex model to address the question of attention allocation at intersections. For the simplified model, AOIs were defined as static areas of the display and for the entire duration of the driving scenario including intersections and non-intersection segments. The AOIs were mutually exclusive and encompassed the entire forward view of the simulator.

For the intersection model, we defined AOIs only in the areas near intersections, not for the long straight driving sections between them. Intersections were divided into seven three-second epochs for analysis. Three epochs were defined before the intersection, one in the intersection , and three were defined following the intersection. AOIs were defined separately for each epoch. 
For instance, if a vehicle was visible in epochs 2, 3, and 4, we defined 3 AOIs for that vehicle. AOIs were marked for each vehicle in the intersection, for traffic signals and signs, and "Looking Ahead". The "Looking Ahead" AOI was assigned the lowest priority in our analysis software so that if any dynamic AOI crossed the "Looking Ahead" AOI, any fixation on that area of overlap was assigned to the dynamic AOI.

Our plan was to use the procedure described in Horrey et al. (2006) to model fixations on the AOIs within each epoch. The weighting for each entity was determined by the formula given in Equation 1 above, substituting the weight (W) for $\mathrm{P}(\mathrm{AOI})$. Where $\mathrm{W}$ is the weight for the vehicles, signal lights, etc. within an intersection, and other parameters are as previously described. In order to simplify the model, we ignored effort by setting it to zero and treated all items as equally salient by setting it to one for all entities. For the simplified model, we ranked relevance, priority, and expectancy using the "lowest ordinal algorithm" (LOA) method (Wickens, et al., 2001). This means that we used the lowest values for the SEEV parameters that maintained the order of the actual values reported by participants in the pilot survey. We assumed that drivers would be fixating on some AOI during the duration of each epoch. The $\mathrm{P}(\mathrm{AOI})$ was calculated as a proportion of the total epoch time.

For the intersection model, we conducted a pilot survey in which we presented participants with static images of the entities to be used in the driving scenarios and asked them to rate each on salience, expectancy and value. All vehicles were presented in the same scene and with the same position and orientation. We assigned rankings for SEEV parameters based on the results of this pilot survey.

Observed data. We used MAPPS 2.1 (EyesDX) to analyze eye tracking data. Based on the marked AOIs, MAPPS produced total fixation times for each AOI. We then calculated P(AOI) as the ratio of the total fixation duration on each AOI to the total duration of the epoch.

Model Fit. We performed a linear regression analysis to fit eye tracking data to the SEEV model. Goodness-of-fit was defined as the $\mathrm{R}^{2}$ value for the regression model.

\section{RESULTS}

\section{Simplified Model}

The simplified model parameters (without Salience or Effort) are presented in Table 2. The model predicted $82 \%$ of total fixation time would be looking down the road (Ahead), $6 \%$ on the dashboard (Dash) and 12\% on other areas (Other). We performed a regression analysis to fit the observed data to the predicted and found that the SEEV model successfully predicted $75 \%$ of the variance in the observed data. Thus we were able to replicate the success of Horrey et al. (2006) in modeling attention with a restricted set of AOIs that are mutually exclusive spatially.

Table 2. Simplified SEEV Model Parameters

\begin{tabular}{|c|c|c|c|}
\hline $\mathrm{AOI}$ & Relevance & Priority & Expectancy \\
\hline Other & 1 & 2 & 2 \\
\hline Dash & 2 & 1 & 1 \\
\hline Ahead & 3 & 3 & 3 \\
\hline
\end{tabular}




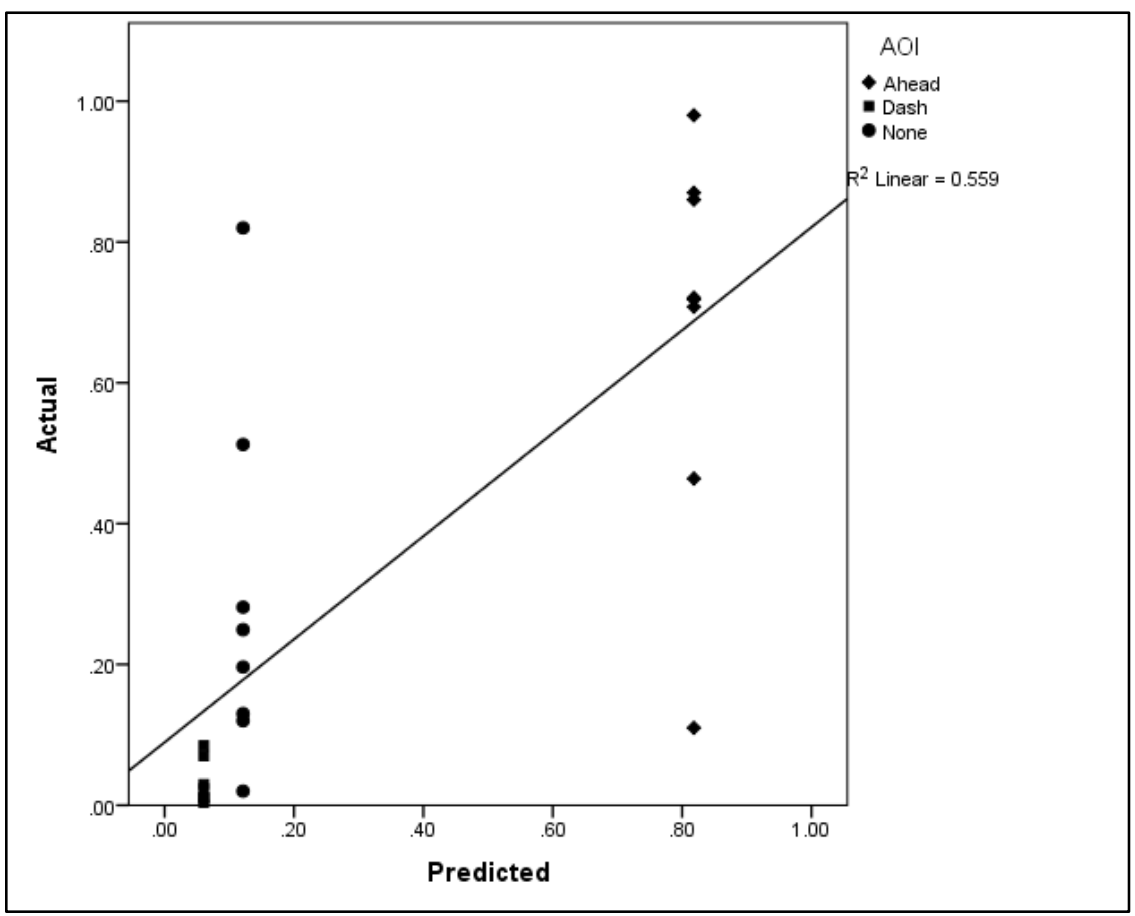

Figure 1. Regression results for fitting the simplified model to the observed data. $\mathbf{N}=8$

\section{Intersection Model}

Results of fitting the Ideal Driver model to 8 participants are presented in Table 4 below. The average $\mathrm{R}^{2}$ value was 0.034 , which by any account is a very poor fit. The present instantiation of the SEEV model does not provide a good prediction of observed scanning behavior among experienced drivers.

Table 3. Sample of Intersection SEEV Model Parameters for one intersection epoch

\begin{tabular}{lrrr}
\hline AOI & Relevance & Priority & \multicolumn{2}{c}{ Expectancy } \\
\hline Ambulance & 1 & 4 & 3 \\
Green Traffic Light & 1 & 3 & 1 \\
Speed Limit Sign & 1 & 1 & 1 \\
Looking Ahead & 2 & 2 & 2 \\
\hline
\end{tabular}




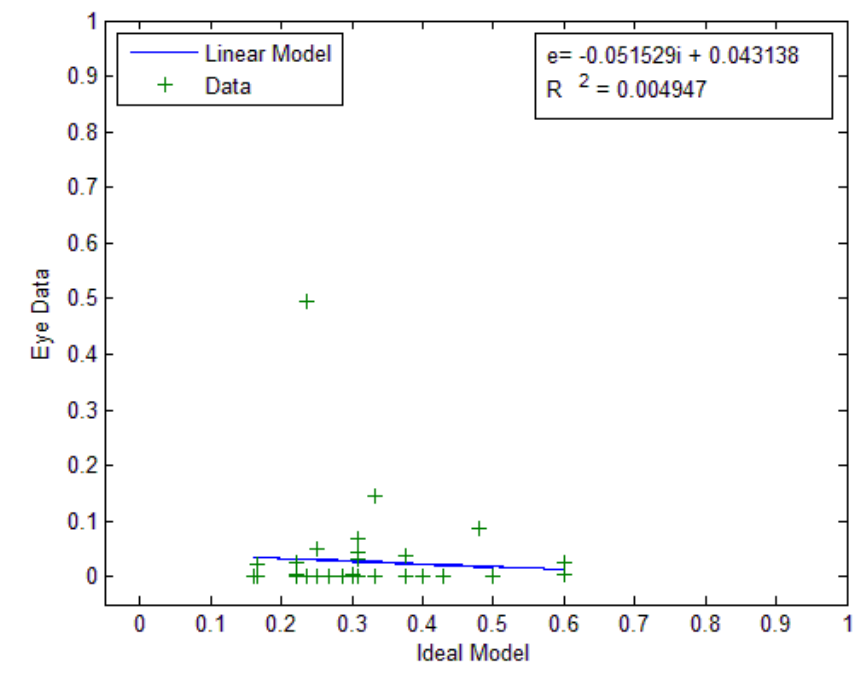

Figure 2. Representative results from one participant for the intersection SEEV model

Figure 2 illustrates a representative participant’s data. It is apparent from inspecting this graph that for the most part, participants did not fixate the AOIs that we predicted they would.

In order to provide a basis for comparison, we generated a random set of SEEV Parameters and re-fit the observed data to the random model. The random SEEV model still proved to be a poor fit with an average $\mathrm{R}^{2}$ of just 0.062 . Mean results from 8 participants are presented in the table below. Whereas Horrey et al. (2006) found that randomization of SEEV parameters resulted in a substantial reduction in fit quality, we obtained similar values for $\mathrm{R}^{2}$.

Table 4. Average Regression Results for two SEEV Models

\begin{tabular}{|c|c|c|c|c|c|}
\hline \multicolumn{3}{|c|}{ Intersection Model } & \multicolumn{3}{|c|}{ Random SEEV Model } \\
\hline Slope & Intercept & $\mathrm{R}^{2}$ & Slope & Intercept & $\mathrm{R}^{2}$ \\
\hline-0.111 & 0.068 & 0.034 & -.0475 & 0.055 & 0.062 \\
\hline
\end{tabular}

\section{DISCUSSION}

Modeling driver attention allocation to non-hazard events at intersections using our instantiation of the SEEV model based on Horrey et al. (2006) proved to be ineffective. Using survey data from a sample of experienced drivers regarding the value and expectancy of entities in the scenario, we were unable to provide a statistically significant fit to the observed eye movement data.

The SEEV model was conceived to model attention allocation to instruments and displays in the aviation domain. The present application is substantially different in domain (driving vs. aviation) and application (model attention to scene elements vs. in-vehicle instruments). Further, rather than using the lowest-ordinal algorithm, we used surveys to obtain drivers' ratings of SEEV parameters for the vehicles we used in the simulator scenario. The results from the model fits using the survey data were no better than using randomly-generated values for the parameters. One reason for the poor model fit may have been that subjective rating ratings for 
parameter values may not be representative of how the rated vehicle holds attention. This may be due to errors associated with attempting to report consciously on cognitive states that are not normally reported (Ericsson \& Simon, 1980).

Most drivers spent little to no time actually fixating on AOIs we defined as potentially important. In retrospect this makes sense as there were no hazard trials included in the scenario. The events we included were events that required only a brief fixation to determine the potential threat level of the moving vehicle. Once the vehicle was observed to be moving in such a way as to not represent a threat, the participant could avoid fixating the vehicle unless some additional information (e.g. a heading change) suggested reassessment.

Future attempts to evaluate the SEEV model for use in predicting attention allocation to the world should include modeling the potential threat level represented by the vehicle or other entity by virtue of its position, heading, speed, or trajectory. That is, a vehicle that appears to be on a collision course will probably attract attention to a far greater extent than a vehicle crossing an intersection that the driver is not yet negotiating.

\section{ACKNOWLEDGEMENTS}

This work was funded through a grant from Toyota Motor Corporation, Japan

\section{REFERENCES}

Cole, B. L. (1972). Visual aspects of road engineering. Paper presented at the Sixth Conference of the Australian Road Research Boards, Canberra, Australia.

Ericsson, K. A., \& Simon, H. A. (1980). Verbal Reports as Data. Psychological Review, 87(3), 215-251.

Horrey, W. J., \& Wickens, C. D. (2006). Examining the impact of cell phone conversations on driving using meta-analytic techniques. Human Factors, 48(1), 196-205.

Horrey, W. J., Wickens, C. D., \& Consalus, K. P. (2006). Modeling Drivers' Visual Attention Allocation While Interacting With In-Vehicle Technologies. Journal of Experimental Psychology: Applied, 12(2), 67-78.

Steelman-Allen, K. S., McCarley, J. S., Wickens, C. D., Sebok, A., \& Bzostek, J. (2009). NSEEV: A Computational Model of Attention and Noticing. Paper presented at the 53rd Annual Meeting of the Human Factors and Ergonomics Society Annual Meeting, San Antonio, TX.

Wickens, C. D., Goh, J., Helleberg, J., Horrey, W. J., \& Talleur, D. A. (2003). Attentional Modeals of Multitask Pilot Performance Using Advanced Display Technology. Human Factors, 45(3), 360-380.

Wickens, C. D., Helleberg, J., Goh, J., Xu, X., \& Horrey, W. J. (2001). Pilot Task Management: Testing an Attentional Expected Value Model of Visual Scanning (pp. 23): Aviation Research Lab, Institute of Aviation. 